\title{
Detection of Non-Cavitated Occlusal Caries with Impedance Spectroscopy and Laser Fluorescence: an In Vitro Study
}

\author{
Diana Mortensen ${ }^{1}$, Katrine Dannemand ${ }^{2}$, Svante Twetman ${ }^{1}$ and Mette Kirstine Keller ${ }^{1, *}$ \\ ${ }^{I}$ Department of Odontology, Section for Cariology \& Endodontics and Pediatric Dentistry \& Clinical Genetics, Faculty \\ of Health Sciences, University of Copenhagen, Denmark \\ ${ }^{2}$ Department of Odontology, Section of Oral Rehabilitation, Faculty of Health and Medical Sciences, University of Co- \\ penhagen, Denmark
}

\begin{abstract}
Objective: To evaluate the performance of an impedance spectroscopy technology for detecting non-cavitated occlusal caries lesions in permanent teeth in vitro. The method was compared with a commonly used laser fluorescence device and validated against histology.

Material and Methodology: A non-cavitated sample of 100 extracted posterior teeth was randomly selected and assessed for caries on enamel and dentin level with aid of CarioScan PRO (ACIS) and DIAGNOdent pen (LF pen) by three examiners. After the measurements, the extension of the lesion was histologically determined as gold standard. Sensitivity, specificity, accuracy and receiver-operating curves were calculated. Intra- and inter-examiner reproducibility was expressed by intra class correlation coefficients.

Results: The histological caries prevalence was $99 \%$ and $41 \%$ exhibited dentin caries. The ACIS technique displayed high specificities but almost negligible sensitivities at readings $>50$. A similar pattern was noted for the LF pen at readings $>30$. The intra- and inter-examiner reproducibility varied between 0.47 and 0.98 and the values were generally lower for the ACIS technique than for the LF pen. The inter-examiner agreement reached excellent levels with both methods.

Conclusions: In vitro, the ACIS technique showed a low ability to disclose occlusal caries lesions in the enamel and/or dentin of non-cavitated permanent molars. However, further in vivo studies of permanent occlusal surfaces are needed to mirror the clinical situation.
\end{abstract}

Keyword: Caries detection, impedance spectroscopy, laser fluorescence, molar teeth.

\section{INTRODUCTION}

Dental caries is still one of the most prevalent diseases affecting humans of all ages [1]. Untreated, it can lead to pain, infection, tooth loss and impaired quality-of-life. In the last decades, a shift in the management of caries has occurred; from the conservative approach "drilling and filling" to preventive strategies, reverse early signs of caries and preserve tooth structure [2]. Therefore, the early detection of non-cavitated enamel demineralization has come into focus as a prerequisite to enable targeted secondary prevention with a combination of self-applied and professional measures. The traditional tool for caries detection is visual-tactile examination, often combined with bitewing radiographs, exhibiting a fair sensitivity and high specificity for frank cavities [3]. In recent years, adjunct methods based on fiberoptics, fluorescence or electrical impedance have been developed but their use and value in everyday practice is,

*Address correspondence to this author at the Department of Odontology, Section for Cariology \& Endodontics and Pediatric Dentistry \& Clinical Genetics, Faculty of Health Sciences, University of Copenhagen, Denmark; Tel: + 45 353-26691; E-mail: mke@sund.ku.dk however, still disputed [4]. A systematic review including 25 reports of moderate or low quality, graded the evidence as limited for laser fluorescence and electric methods with sensitivities and specificities ranging around $70-80 \%$ [5]. Since then, a new device (CarieScan PRO) based on AC impedance spectroscopy has been launched for clinical use. The method is based on the principle that a low voltage current is directed through the tooth to evaluate mineral density $[6,7]$ and a pilot study has reported good accuracy for precavitated lesions with no false positive outcomes [8]. To our knowledge, an extended evaluation of the method is so far only available for primary teeth [9]. The aim of this in vitro study was therefore to validate occlusal caries detection of permanent molars with AC impedance spectroscopy (CariesScan PRO) against histological examination. As a further comparison, a commonly used device based on laser fluorescence, the DIAGNOdent pen, was included.

\section{MATERIALS AND METHODOLOGY}

\section{Sample Selection}

A random sample of 100 extracted non-cavitated permanent molars was selected from a bank of teeth at the Section 
for Cariology, Department of Odontology at the University of Copenhagen. All molar teeth were visually inspected after drying and were characterized as without any obvious caries lesions or hypomineralized area on the occlusal surfaces. The root surfaces were cleaned with ultrasonic technology to remove tissue remnants, whereas debris located on occlusal surfaces was removed using rotating brushes. The teeth were then given a unique ID-number and stored individually in an airtight plastic cup containing water with $0.1 \%$ thymol in order to avoid bacterial growth. The sample size was determined on the basis of suggested inclusion criteria in a comprehensive systematic review on adjunct methods for caries detection [5].

\section{Caries Assessment}

An "occlusal map", consisting of standardized printed occlusal surface was constructed for registration of a unique test site associated to each ID number. The first examiner marked the occlusal fissure site of interest. Each tooth was examined in a randomized order with the ACIS and LF techniques described below by three independent dentists at two separate occasions with a minimal interval of one week. Before the study, the examiners were familiarized with both caries detection systems and trained themselves with a pilot material of 100 extracted molars selected with identical inclusion criteria. The examiners were blinded for the peer's scores.

\section{Alternating Current Impedance Spectroscopy (ACIS) Readings}

The CarieScan PRO (CarieScan Ltd, Dundee, Scotland) technology was used for the ACIS assessments, calibrated and handled according to the manufacturer's manual. Each tooth was rinsed in tap water for 5 seconds and then gently dried at the crown area for 3 seconds by an air-syringe, leaving the root surface wet. The teeth were held by the roots with two moist fingers in the same ungloved hand (left) as the lip hook connector and the CarieScan PRO device was held by a glove-covered hand (right). The ends of the sensor tip were pressed into the fissures with the same light pressure as when using a pen, ensuring that the tip was not moving during measurement. The test value was registered when the device three times showed a stable score at the preselected site.

\section{Laser Fluorescence (LF) Readings}

The DIAGNOdent pen (KaVo, Biberach, Germany) with a cylindrical probe was used for the laser fluorescence readings. Before each series of measurement, the pen was calibrated with a ceramic standard as recommended in the manufacturer's manual. Each tooth was dried with compressed air for 5 seconds immediately before the recording. The probe was first placed at an obviously healthy part of the buccal area to register the fluorescence of sound enamel, later to be subtracted from the peak value. The preselected test site was thereafter evaluated through pivoting in all directions and the highest value was recorded.

\section{Histological Validation}

After the final examination, the teeth were submitted for hemi section for obtaining the gold standard. The cutting was performed with aid of an EXAKT band saw (band size 0.27 $\mathrm{mm}$; Norderstedt, Germany) through the chosen test site in mesio-distal direction. Both sides of each tooth were inspected under a stereomicroscope (Zeiss, Germany) and the most profound caries stage was registered. Carious demineralization was defined as opaque or brownish discoloration and the extent of the lesion was categorized in five groups according to Downer [10]; D0 = caries-free; D1 = caries reaching the outer half of the enamel; D2 = caries in the inner half of the enamel; D3 = caries in the outer half of the dentin, and, D4 = caries in the inner half of the dentin. Two examiners carried out this evaluation independently, at least two weeks after the ACIS and LF pen readings, to obviate examiner bias. When findings showed an inter-examiner discrepancy, a reevaluation was done until consensus was achieved. From these data, the true prevalence of caries in the studied selection was calculated.

\section{Statistical Analyses}

All data were processed with the IBM SSPS software (version 20.0, Chicago, Ill, USA). Readings in relation to caries extension were tested with t-tests. The reproducibility of the DIAGNOdent pen and CarieScan PRO were measured using interclass correlation coefficients (ICC). Sensitivity, specificity, accuracy and area under the ROC-curve were calculated at D2 and D3 levels with different cut-off points. For enamel and dentin caries, ACIS cut-off values ranging from 40 to 90 were computed while the corresponding readings for the LF pen ranged between 10 and 40 .

\section{RESULTS}

The histological examination revealed a caries prevalence of $99 \%$; only one tooth was caries free. Twenty five teeth had caries extending halfway through the enamel (D1), 33 teeth had caries in the inner half of the enamel (D2) and $41 \%$ exhibited dentin caries (D3+D4). The mean readings of the ACIS and the LF pen according to this classification are shown in Table $\mathbf{1}$. The extension of the carious lesion was associated with increasing ACIS readings and the differences between D2 and D3 vs. D1 were statistically significant $(p<0.05)$. No similar tendencies were however unveiled with the LF pen.

The in vitro validity of the two methods to detect occlusal caries on enamel (D2) and dentin (D3) level is summarized in Tables $\mathbf{2}$ and 3. The ACIS method showed a high specificity at values over 50 but a poor sensitivity. A similar pattern was shown with the LF pen concerning readings $>30$. The accuracy was generally modest and varied between 0.52 and 0.70 for both methods. For the ACIS technique, the combination of sensitivity $(0.76)$ and specificity $(0.54)$ that would maximize Youden's Index [11] for enamel lesions was evident for readings around 40 . Accuracy at this point was 0.70 . The area under the ROC curve was $<0.65$ for all combinations with both methods. 
Table 1. Mean values (SD) of readings in relation to the Downer classification [Downer].

\begin{tabular}{|c|c|c|c|c|}
\hline & D1 $(\mathbf{n}=\mathbf{2 5})$ & D2 $(\mathbf{n}=\mathbf{3 3})$ & D3 $(\mathbf{n}=\mathbf{3 3})$ & D4 $(\mathbf{n}=\mathbf{8})$ \\
\hline \hline ACIS & $33.1(20.3)$ & $43.9(26.3)$ & $46.0 *(23.9)$ & $52.8 *(15.1)$ \\
\hline LF pen & $13.8(19.5)$ & $16.7(23.2)$ & $16.5(20.6)$ & $11.8(10.8)$ \\
\hline
\end{tabular}

* Statistically significance difference compared with D1 $(\mathrm{p}<0.05)$

Table 2. Validity of AC impedance spectroscopy (ACIS, CarieScan PRO) and laser fluorescence (DIAGNOdent pen) in detecting occlusal caries on dentin (D2) level. Values in the table are based on the median of three independent examiners.

\begin{tabular}{|c|c|c|c|c|c|}
\hline Method & Cut-Off Reading & Sensitivity & Specificity & Accuracy & Area Under ROC \\
\hline ACIS & 40 & 0.76 & 0.54 & 0.70 & 0.64 \\
\hline ACIS & 70 & 0.12 & 1.00 & 0.35 & 0.56 \\
\hline ACIS & 90 & 0.08 & 1.00 & 0.32 & 0.54 \\
\hline LF pen & 20 & 0.23 & 0.89 & 0.40 & 0.56 \\
\hline LF pen & 40 & 0.08 & 0.96 & 0.31 & 0.52 \\
\hline
\end{tabular}

Table 3. Validity of AC impedance spectroscopy (ACIS, CarieScan Pro) and laser fluorescence (DIAGNOdent pen) in detecting occlusal caries on dentin (D3) level. Values in the table are based on the median of three independent examiners.

\begin{tabular}{|c|c|c|c|c|c|}
\hline Method & Cut-Off Reading & Sensitivity & Specificity & Accuracy & 0.55 \\
\hline \hline ACIS & 40 & 0.78 & 0.39 & 0,64 & 0.59 \\
\hline ACIS & 50 & 0.39 & 0.81 & 0.60 & 0.60 \\
\hline ACIS & 70 & 0.12 & 0.93 & 0.60 & 0.53 \\
\hline ACIS & 90 & 0.05 & 0.98 & 0.49 \\
\hline LF pen & 10 & 0.49 & 0.83 & 0.59 & 0.52 \\
\hline LF pen & 20 & 0.24 & 0.93 & 0.58 & 0.50 \\
\hline LF pen & 40 & 0.07 & & 0.54 \\
\hline
\end{tabular}

The intra- and inter-examiner reproducibility is presented in Table 4. The inter class correlation values varied between 0.47 and 0.98 and were generally lower for the ACIS technique than those obtained with the LF pen. Furthermore, the inter-examiner reproducibility was stronger than the intraexaminer values for both methods, reaching excellent levels of agreement.

\section{DISCUSSION}

The aim of this study was to investigate the ability of one new and one established technique to detect occlusal noncavitated caries lesions from a clinician's point of view who ideally wants an aid to decide between secondary prevention and restorative treatment. Thus, the main focus was on detecting non-cavitated lesions within the dentin rather than lesions restricted to the enamel. The clear-cut and discouraging finding was that both methods performed less good for this purpose. When the recommended cut-off levels for restorative therapy (high probability of caries) were applied ( $>90$ and $>30$ for the ACIS and LF pen, respectively), high specificities $(<0.90)$ were achieved but an almost negligible sensitivity. The low sensitivity indicated a high risk that dentin lesions may remain undetected and overlooked; on the other hand, the risk for restorative overtreatment was low. Interestingly, the clinical performance was not significantly improved when the detection level was moved to the inner enamel level. Unfortunately, the present study sample did not allow for an analysis concerning the differentiation between sound and demineralized fissures. 
Table 4. Intra- and inter-examiner reproducibility using laser fluorescence (LF pen) and AC impedance spectroscopy (ACIS). Values in table denote the mean ICC value and $95 \%$ CI.

\begin{tabular}{|c|c|c|}
\hline & LF Pen & ACIS \\
\hline \hline Examiner 1 & $0.95(0.93-0.97)$ & $0.79(0.70-0.85)$ \\
\hline Examiner 2 & $0.90(0.86-0.94)$ & $0.64(0.51-0.74)$ \\
\hline Examiner 3 & $0.87(0.81-0.91)$ & $0.47(0.30-0.61)$ \\
\hline Examiner 1 vs. 2 & $0.98(0.97-0.99)$ & $0.81(0.71-0.87)$ \\
\hline Examiner 1 vs. 3 & $0.98(0.97-0.99)$ & $0.78(0.68-0.75)$ \\
\hline Examiner 2 vs. 3 & $0.98(0.97-0.99)$ & $0.89)$ \\
\hline
\end{tabular}

Our findings of poor performance with the ACIS method were in agreement with the recent report Teo and coworkers [9]. Based on their findings, in vitro as well as in vivo, it was concluded that the CarieScan PRO currently was not suitable for the use in the primary dentition and permanent molars have both deeper and more complex fissures than deciduous teeth. The suboptimal function of the LF pen was however more surprising since a body of literature has reported a good performance for occlusal caries detection with that method [12-14]. Nevertheless, the common recommendation is that fluorescence-based methods may be restricted to provide a second opinion after visual-tactile inspection and radiographic examination in clinical practice $[5,15]$.

The results obtained from the present study must however be considered with great caution and a number of limitations must be considered. First of all, findings from in-vitro studies can never directly reflect the real-life situation and a previous study have indicated a poor agreement between outcome measures in vitro and in vivo [9]. Secondly, all teeth but one were decayed and a "normal" and even distribution of sound teeth would certainly have affected the sensitivity and specificity values. Thirdly, the storage media, temperature and the applied methodology may have impaired the performance of both methods [16]. For example, the drying time is crucial for the ACIS method and it is possible that the extracted teeth in our experiment were somewhat over-dried with impaired conductivity. The thymol-containing storage media together with the mechanical fissure cleaning is somewhat detrimental for the LF method since the device detects biological luminescence emitted by bacteria and its metabolites [17].

The different (moderate to strong) intra-examiner reproducibility among the three dentists with the ACIS technique was notable. The method was new to all of them but they shared the same run-in training period containing readings of at least one-hundred occlusal surfaces before entering this study. The intra class correlation was however clearly in line with previous reports [9], although inferior to those reported with the LF pen $[9,12]$. This may indicate that the wider sensor tip of the ACIS device was less distinct to apply and to handle steadily in fissures when compared with the narrower tip of the LF pen.

\section{CONCLUSION}

Within the limitations of this in vitro study, it was concluded that ACIS technique displayed a low ability to disclose occlusal caries lesions in the enamel and/or dentin in non-cavitated permanent molars. To more adequately mirror the clinical situation, further in vivo studies of occlusal caries detection are needed, since treatment of fissure caries constitute a major challenge in clinical decision-making.

\section{CONFLICT OF INTEREST}

The authors confirm that this article content has no conflicts of interest.

\section{ACKNOWLEDGEMENT}

Declared none.

\section{REFERENCES}

[1] Selwitz RH, Ismail AI, Pitts NB. Dental caries. Lancet 2007; 369(9555): 51-9.

[2] Pitts NB. Are we ready to move from operative to nonoperative/preventive treatment of dental caries in clinical practice? Caries Res 2004; 38(3): 294-304.

[3] Neuhaus KW, Longbottom C, Ellwood R, Lussi A. Novel lesion detection aids. Monogr Oral Sci 2009; 21: 52-62.

[4] Souza JF, Boldieri T, Diniz MB, Rodrigues JA, Lussi A, Cordeiro RC. Traditional and novel methods for occlusal caries detection: performance on primary teeth. Lasers 2013; 28(1): 287-95.

[5] Twetman S, Axelsson S, Dahlen G, et al. Adjunct methods for caries detection: A systematic review of literature. Acta Odontol Scand 2013;71(3-4): 388-97.

[6] Huysmans MC, Longbottom C, Pitts NB, Los P, Bruce PG. Impedance spectroscopy of teeth with and without approximal caries lesions: an in vitro study. J Dent Res 1996; 75(11): 1871-8.

[7] Longbottom C, Huysmans MC, Pitts NB, Los P, Bruce PG. Detection of dental decay and its extent using a.c. impedance spectroscopy. Nat Med 1996; 2(2): 235-7.

[8] Pitts NB, Longbottom C, Hall AF. Diagnostic accuracy of an optimized ac impedance device to aid caries detection and monitoring. Caries Res 2008; 42(3): 211

[9] Teo TK, Ashley PF, Louca C. An in vivo and in vitro investigation of the use of ICDAS, DIAGNOdent pen and CarieScan PRO for the detection and assessment of occlusal caries in primary molar teeth. Clin Oral Investig 2013. [Epub ahead of print]

[10] Downer MC. Concurrent validity of an epidemiological diagnostic system for caries with the histological appearance of extracted teeth as validating criterion. Caries Res 1975; 9(3): 231-46. 
[11] Youden WJ. Index for rating diagnostic tests. Cancer 1950; 3: 32-5.

[12] Rodrigues Jde A, Hug I, Diniz MB, Cordeiro RC, Lussi A. The influence of zero-value subtraction on the performance of two laser fluorescence devices for detecting occlusal caries in vitro. J Am Dent Assoc (1939) 2008; 139(8): 1105-12.

[13] Diniz MB, Boldieri T, Rodrigues JA, Santos-Pinto L, Lussi A, Cordeiro RC. The performance of conventional and fluorescence-based methods for occlusal caries detection: an in vivo study with histologic validation. J Am Dent Assoc 2012; 143(4): 339-50.

[14] Achilleos EE, Rahiotis C, Kakaboura A, Vougiouklakis G. Evaluation of a new fluorescence-based device in the detection of incipient occlusal caries lesions. Lasers Med Sci 2013; 28(1): 193-201.
[15] Gomez J, Zakian C, Salsone S, et al. In vitro performance of different methods in detecting occlusal caries lesions. J Dentist 2013; 41(2): 180-6.

[16] Francescut P, Zimmerli B, Lussi A. Influence of different storage methods on laser fluorescence values: a two-year study. Caries Res 2006; 40(3): 181-5.

[17] Lussi A, Hellwig E. Performance of a new laser fluorescence device for the detection of occlusal caries in vitro. J Dentist 2006; 34(7): 467-71.

Received: December 30, 2013

Revised: February 10, 2014

Accepted: February 11, 2014

(C) Mortensen et al.; Licensee Bentham Open.

This is an open access article licensed under the terms of the Creative Commons Attribution Non-Commercial License (http://creativecommons.org/licenses/by-nc/3.0/) which permits unrestricted, non-commercial use, distribution and reproduction in any medium, provided the work is properly cited. 